\title{
Autosomal dominant burning feet syndrome
}

Florian Stögbauer, Peter Young, Gregor Kuhlenbäumer, Reinhard Kiefer, Vincent Timmerman, E Bernd Ringelstein, Jian-Feng Wang, J Michael Schröder, Christine Van Broeckhoven, Joachim Weis

Department of

Neurology, University of Münster, Germany F Stögbauer

G Kuhlenbäumer

P Young

R Kiefer

E B Ringelstein

Department of

Neuropathology,

University of Aachen,

Germany

J-F Wang

J M Schröder

Institute of Pathology, University of Bern, Switzerland

$\mathrm{J}$ Weis

Neurogenetics Laboratory, Flanders Interuniversity

Institute for Biotechnology (VIB), Born Bunge

Foundation (BBS), Department of Biochemistry,

University of Antwerp (UIA), Belgium

G Kuhlenbäumer

V Timmerman

C Van Broeckhoven

Correspondence to: Dr Florian Stögbauer, Department of Neurology,

University of Münster,

Albert Schweitzer Strasse 33,

D-48129 Münster, Germany.

Telephone 0049251

8348178; fax 0049251

8348181; email

stogbau@uni-muenster.de

Received 29 May 1998 and in final form

27 January 1999

Accepted 4 February 1999

\begin{abstract}
Familial burning feet syndrome inherited as an autosomal dominant trait has been described in only one family. Due to an associated sensory neuropathy the autosomal dominant burning feet syndrome was suggested to represent a variant form of hereditary sensory and autonomic neuropathy type I (HSAN I). Clinical, histopathological, and molecular genetic studies were performed in a large German kindred with autosomal dominant burning feet syndrome. The autosomal dominant burning feet syndrome was associated with a neuropathy predominantly affecting small unmyelinated nerve fibres. Linkage to the HSAN I locus on chromosome 9q22 and to the CharcotMarie-Tooth disease type 2B (CMT 2B) locus on chromosome 3q13-q22 was excluded. The autosomal dominant burning feet syndrome is neither allelic to HSAN I nor to CMT 2B and thus represents a distinct genetic entity.
\end{abstract}

(F Neurol Neurosurg Psychiatry 1999;67:78-81)

Keywords: burning feet syndrome: hereditary sensory neuropathy

Burning feet syndrome is characterised by burning, tingling, numbness, and a dull ache in the feet and legs. Symptoms are worsened by heat and ameliorated by cooling. Several causes have been described, including vitamin deficiency and poor nutritional status. ${ }^{1-3}$ Burning feet were occasionally found to be associated with tarsal tunnel syndrome and gastric surgery. ${ }^{4}$

To date, only one family has been reported in which burning feet were the only clinical manifestation of a dominantly inherited sensory neuropathy. ${ }^{6}$ In this family clinical and neurophysiological studies of affected members showed only mild abnormalities but in sural nerve biopsies increased frequencies of demyelinating and remyelinating teased fibres were found. The authors concluded that the inherited form of burning feet syndrome might represent a different type of hereditary sensory and autonomic neuropathy type I (HSAN I) or a sensory neuropathy distinct from HSAN I.

Hereditary sensory and autonomic neuropathies represent a heterogenous group of disor- ders of sensory and autonomic nerves. Six subtypes of HSAN were classified $^{7}$; HSAN I typically presents as a distal lower limb sensory and autonomic neuropathy with skin injuries sometimes leading to acral mutilation. Clinical features may also include lancinating pain, muscle weakness, sensory ataxia, spasticity, and neurogenic arthropathy. Whether this clinical heterogeneity represents defects in different genes or different alleles of the same gene is not known.

Recently, the chromosomal locus for HSAN I was mapped to chromosome 9q22.1-q22.3. The chromosomal locus of another autosomal dominant neuropathy with prominent sensory abnormalities, Charcot-Marie-Tooth disease type 2B (CMT 2B), was mapped to chromosome 3q13-q22. ${ }^{9-11}$ As there are overlaps in the phenotype of HSAN I and CMT 2B it has been proposed that CMT 2B should rather be classified as HSAN I, implying multiple gene loci for this syndrome. ${ }^{12}$

In the present report, we describe another family in which the burning feet syndrome was inherited as an autosomal dominant trait. Clinical, neurophysiological, histopathological, and molecular genetic studies were performed.

\section{Patients and methods}

A German kindred was diagnosed with autosomal dominant burning feet syndrome. Sixty eight family members including 10 affected members were available for this study (fig 1). Twenty seven members including all patients underwent neurological examination and 20 members were used in the linkage studies.

Nerve conduction studies and needle EMG were performed according to standard techniques. Three affected members of the family including the propositus were investigated (III:31, 49 years; III:33, 55 years; IV:30, 27 years).

A sural nerve biopsy of the index patient (III:31) was examined by light and electron microscopy as described elsewhere..$^{13}$ Semithin cross sections of 455 randomly selected myelinated nerve fibres from several fascicles were stained with toluidine blue and evaluated morphometrically using a digital image analysis system (Kontron KS 300, Munich, Germany).

Short tandem repeat (STR) analysis was performed with the chromosome 9q22.1q22.3 markers D9S257, D9S196, D9S197, 


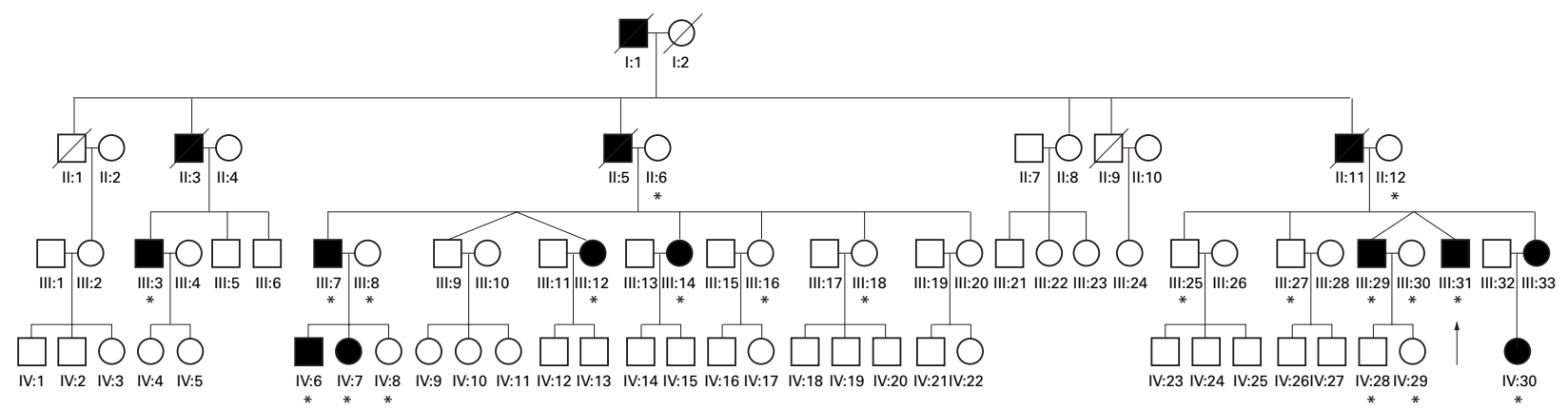

Figure 1 Pedigree of the German family with autosomal dominant burning feet syndrome. Slashed=deceased; squares=males; circles=females; filled=affected members; empty=non-affected members. The propositus is marked with an arrow. Those that were used in linkage studies are marked by an asterix.

D9S280, D9S910, and the chromosome 3q13q22 markers D3S1769, D3S1551, D3S1290, and D3S1744 as described. ${ }^{14-16}$ Two point linkage analysis was performed with the FASTLINK computer package. ${ }^{17}$ We assumed that the disease is transmitted as a fully penetrant autosomal dominant trait with a gene frequency of $1 / 10.000$. Allele frequencies of the dinucleotide repeat markers were obtained from the Genome Data Base (www.gdb.org). The allele frequencies of the markers D9S910, D3S1769, and D3S1744 are not known. Therefore we used equal allele frequencies for these markers. Haplotypes were constructed according to the genetic map of Dib et $a l,{ }^{15}$ and the Cooperative Human Linkage Center (www.chcl.org).

\section{Results}

The burning feet syndrome was inherited as an autosomal dominant trait (fig 1). The onset of symptoms ranged between 12 and 40 years (median 26.8). The affected family members showed a homogeneous phenotype. Pain was the main symptom and present in all affected members. They complained of symmetric burning and dull aching pain in the feet, predominantly affecting the soles and toes with progressive involvement of the ankles and lower legs. Symptoms did not occur in the hands and forearms, worsened when the feet were warm, and were ameliorated by cooling. There were no complaints of weakness or clinical signs of autonomic disturbances.

On neurological examination, no muscular atrophy or muscle weakness was found in the affected family members, and tendon reflexes were normal. None of the patients had high arched feet, pes cavus, or other skeletal abnormalities. Touch, pain, temperature, and vibration sensation as well as position sense were normal.

In the index patient (III:31), nerve conduction studies of the right tibial nerve showed a slightly reduced amplitude of the compound muscle action potential of the extensor digitorum brevis muscle and axon reflexes in $\mathrm{F}$ wave recordings. The distal motor latency, nerve conduction velocity, and $\mathrm{F}$ wave latency were normal. Motor nerve conduction and $\mathrm{F}$ wave studies of the right median and ulnar nerve were normal. Sensory nerve conduction studies of the right median, ulnar, and sural nerve were normal except for a slight reduction in amplitude of the ulnar sensory nerve action potential, with a normal amplitude on the opposite side. On EMG of the right tibialis anterior muscle, no pathological spontaneous activity was detected. However, recruitment of motor units was reduced and the mean amplitude of motor unit action potentials was increased, compatible with a mild chronic neurogenic process. Nerve conduction studies and EMG of III:33 were normal except for A waves present on $\mathrm{F}$ wave recordings from the right tibial nerve. Electrophysiological testing of IV:30 gave normal results.

On sural nerve biopsy (III:31) there was moderate reduction of myelinated nerve fibres, especially of the smaller ones, which was partially compensated by regenerated fibres (fig 2 A). Repeatedly, myelin debris, small clusters of regenerated myelinated nerve fibres, and disproportionately thin myelin sheaths around large axons were found. Most strikingly, however, a marked reduction of unmyelinated nerve fibres was found by electron microscopy. Numerous collagen pockets indicating loss of unmyelinated nerve fibres and Schwann cell processes without any association with axons were seen (fig 2 B). The morphometrical analysis of myelin sheaths and axonal areas disclosed that the total number of myelinated nerve fibres per endoneural area was just below the lower normal range $\left(5912 / \mathrm{mm}^{2}\right.$; normal range $6000-9000 / \mathrm{mm}^{2}$ ) due to an increase of small regenerated nerve fibres. Accordingly the myelin area per endoneural area was only moderately reduced $(17.9 \%$; normal range $20 \%-30 \%)$. At the electron microscopic level, there were 167 empty Schwann cell processes per 231 unmyelinated axons and 68 myelinated nerve fibres as counted on 32 electron migrographs (total area evaluated: $0.0076 \mathrm{~mm}^{2}$ ), indicating loss of unmyelinated nerve fibres.

Genetic linkage was performed with STR markers that cover the complete HSAN I candidate region on chromosome 9q22.1-q22.3. Haplotype analysis showed multiple recombinants and significant negative lod scores were obtained with all markers. Haplotype analysis of the CMT 2B locus on chromosome 3q13-q22 also showed multiple recombinants. Significant negative lod scores over the whole region were obtained. Thus, the autosomal 

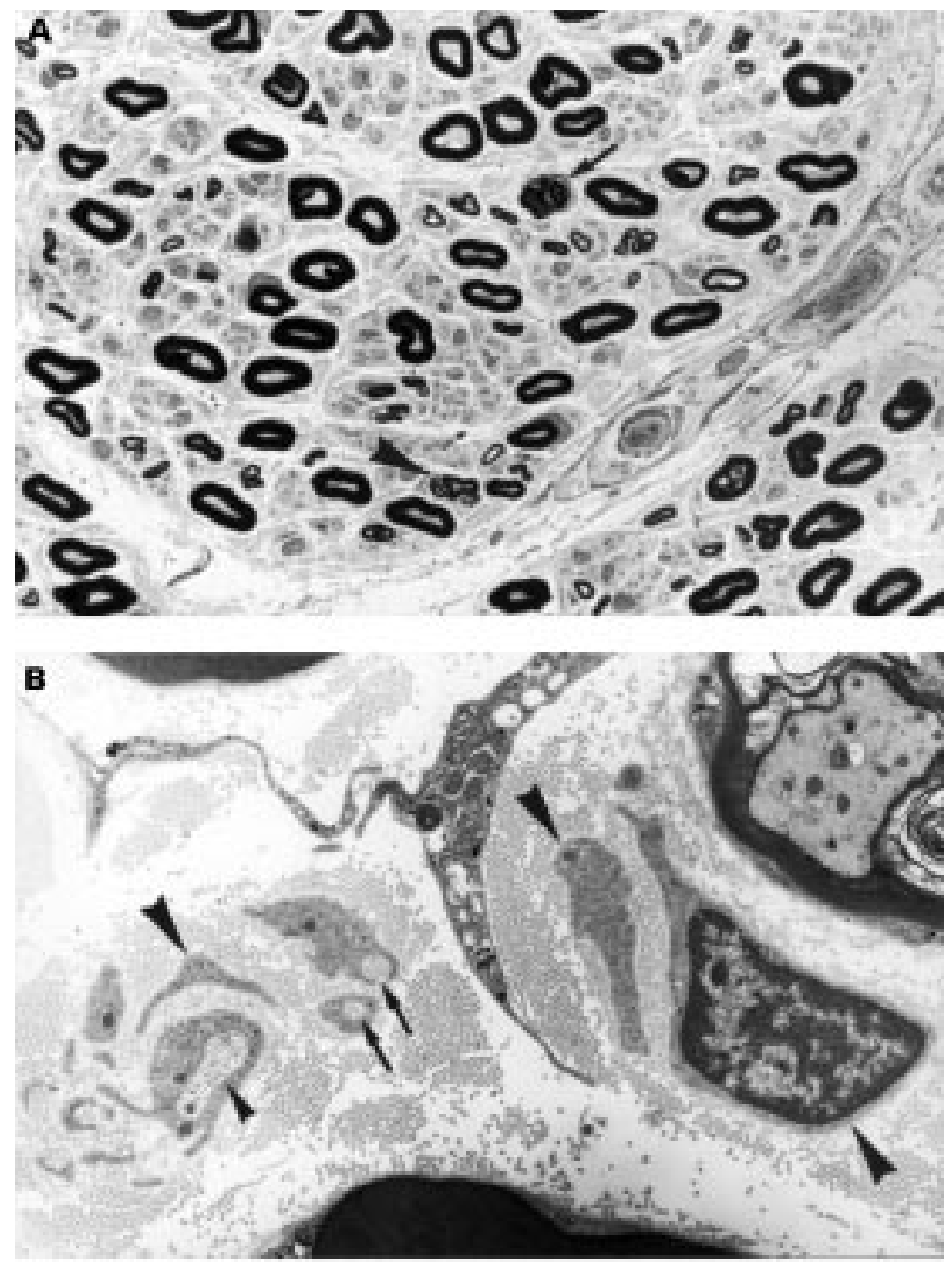

Figure 2 (A) Occasional degenerating myelinated fibres (arrow), myelin breakdown products, and small clusters of regenerating nerve fibres (arrowhead) were found in transverse semithin sections of the sural nerve. Epon, paraphenylendiamine (originally $\times 400$ ). (B) On electron microscopy, bundles of collagen fibres are seen surrounded by Schwann cell processes ("collagen pockets"; arrows), indicating preceeding degeneration of unmyelinated axons. Isolated Schwann cells and stacks of Schwann cell processes (bands of Buenger) at the site of degenerated myelinated fibres are also apparent (large arrowheads). A preserved unmyelinated axon is indicated by a small arrowhead (originally $\times 23000$ ). ological examination there were minor abnormalities on motor nerve conduction studies and EMG in two patients. These data point towards a mild axonal neuropathic process involving motor nerves. However, the changes are very mild and not necessarily related to burning feet syndrome as such minor abnormalities are often found in older patients due to subclinical motor root involvement after degenerative spinal disease. Histopathologically, the myelinated nerve fibre density was moderately reduced. Most notably, however, there was a marked reduction in the density of unmyelinated nerve fibres. This reduction might reflect predominant involvement of this fibre population. Similar findings have been described in HSAN and are in line with the above mentioned abnormalities of small fibre function in burning feet syndrome. ${ }^{718}$

Clinical as well as histopathological data on the families described suggest that the autosomal dominant burning feet syndrome is the main manifestation of a primarily sensory neuropathy with predominant involvement of unmyelinated fibres. With regard to the age of onset of the disease and the preferential involvement of the lower limbs it was proposed that the burning feet syndrome may represent one of the variant forms of HSAN type $I$ in which sensory disturbances (lancinating pain or burning feet) are the only signs of a sensory neuropathy. ${ }^{6}$

Recently, the chromosomal locus for HSAN I has been mapped to chromosome 9q22.1-q22.3. ${ }^{8}$ Molecular genetic analysis excludes the HSAN I locus in our family. CMT 2B has been mapped to chromosome $3 q 13-q 22 .{ }^{10}{ }^{11}$ Along with minor motor nerve involvement, sensory symptoms are prominent in this neuropathy. Patients often have distal sensory loss and non-healing ulcerations sometimes leading to amputations. Because of this overlap of symptoms it has been suggested that the disease condition is misclassified and represents more likely a subtype of HSAN I. ${ }^{12}$ The sensory symptoms in the family we describe are milder but it is not uncommon that different mutations in the same gene may lead to phenotypes of different severities. For example, different mutations in the myelin protein zero gene can lead to a wide range of phenotypes. ${ }^{19}$ Nevertheless, molecular genetic analysis excludes the CMT type $2 \mathrm{~B}$ locus as well.

In conclusion, our results confirm the presence of a predominantly sensory neuropathy in autosomal dominant burning feet syndrome. The results of the molecular genetic studies exclude linkage to both known loci of inherited sensory neuropathies (HSAN I and CMT 2B). Thus, the autosomal dominant burning feet syndrome most likely represents a separate disease entity. objective clinical evidence of the underlying neuropathy. However, quantitative psychophysical studies to investigate pain and temperature thresholds could not be performed. Others have found that these are abnormal in a subpopulation of patients with burning feet syndrome possibly indicating involvement of small unmyelinated fibres. ${ }^{18}$ On neurophysi-
F Stögbour and P Young contributed equally to the work presented in this article.

We are grateful to the patients and their relatives for their kind cooperation in our research project and to Mrs A Knischewski, Mrs E Beck, Mrs E De Vriendt, and Mrs H Mader for technical assistance. This work was supported in part by a grant from the assistance. This work was supported in part by a grant from the
Fund for Scientific Research (FWO), Flanders-Belgium. VT is a research assistant of the FWO, Belgium. GK is supported by 
the Deutsche Forschungsgemeinschaft (KU 1194/1-1). CVB is the coordinator of the European CMT Consortium sponsored by an EU BIOMED2 grant (CT 96-1614).

1 Skelton WP, Skelton NK. Thiamine deficiency neuropathy. It is still common today. Postgrad Med 1989;85:301-6.

2 Grande Covian F. Vitamin deficiencies during the Spanish Civil War in Madrid: a reminiscence. Acta Vitaminologia Enzymologica (Milano) 1982;4:99-103.

3 Lai CS, Ransome GA. Burning-feet syndrome. Case due to malabsorption and responding to riboflavine. BMF 1970;2: $151-2$

4 Webb J, March L. Tarsal tunnel syndrome and burning feet. Med $\mathcal{F}$ Aust 1987;147:311-2.

5 Abarbanel JM, Berginer VM, Osimani A, et al. Neurologic complications after gastric restriction surgery for morbid obesity. Neurology 1987;37:196-200.

6 Dyck PJ, Low PA, Stevens JC. Burning feet as the only manifestation of dominantly inherited sensory neuropathy. Mayo Clin Proc 1983;58:426-9.

7 Dyck PJ. Neuronal atrophy and degeneration predominantly affecting peripheral sensory and autonomic neurons. In: Dyck PJ, Thomas PK, Griffin JW, et al, eds. Peripheral neuropathy. Philadelphia: Saunders, 1993:1065Periphe

8 Nicho

Nicholson GA, Dawkins JL, Blair IP, et al. The gene for hereditary sensory neuropathy type I (HSN-I) maps to chromosome 9q22.1-q22.3. Nat Genet 1996;13:101-4.

9 Elliott JL, Kwon JM, Goodfellow PJ, et al. Hereditary motor and sensory neuropathy IIB: clinical and electrodiagnostic characteristics. Neurology 1997;48:23-8.

10 Kwon JM, Elliott JL, Yee WC, et al. Assignment of a second Charcot-Marie-Tooth type II locus to chromosome 3q. Am f Hum Genet 1995;57:853-8.
11 De Jonghe P, Timmerman V, FitzPatrick D, et al. Mutilating neuropathic ulcerations in a chromosome 3q13-q22 linked Charcot-Marie-Tooth disease type 2B family. $\mathcal{F}$ Neurol Neurosurg Psychiatry 1997;62:570-3.

12 Vance JM, Speer MC, Stajich JM, et al. Misclassification and linkage of hereditary sensory and autonomic neuropathy type 1 as Charcot-Marie-Tooth disease, type 2B. Am 7 Hum Genet 1996;59:258-62.

13 Schröder JM, Mayer M, Weis J. Mitochondrial abnormalities and intrafamilial variability of sural nerve biopsy findings in adrenomyeloneuropathy. Acta Neuropathol 1996;92: 64-9.

14 Sheffield VC, Weber JL, Buetow KH, et al. A collection of tri- and tetranucleotide repeat markers used to generate high quality, high resolution human genome-wide linkage maps. Hum Mol Genet 1995;4:1837-44.

15 Dib C, Faure S, Fizames C, et al. A comprehensive genetic map of the human genome based on 5.264 microsatellites. Nature 1996;380:152-4.

16 Stögbauer F, Young P, Timmerman V, et al. Refinement of the hereditary neuralgic amyotrophy (HNA) locus to chromosome 17q24-q25. Hum Genet 1997;99,685-7.

17 Cottingham RJ, Idury R, Schaffer A. Faster sequential genetic linkage computations. Am f Hum Genet 1993;53: $252-63$

18 Smith SJM, Ali Z, Fowler CJ. Cutaneous thermal thresholds in patients with painful burning feet. $f$ Neurol Neurosurg Psychiatry 1991;54:877-81.

19 Warner LE, Hilz MJ, Appel SH, et al. Clinical phenotypes of different MPZ (P0) mutations may include CharcotMarie-Tooth type 1B, Dejerine-Sottas, and congenital hypomyelination. Neuron 1996;17:451-60. 